\title{
Scrambling in spoken Dutch: Definiteness versus weight as determinants of word order variation
}

\author{
GEERTJE VAN BERGEN and PETER DE SWART
}

\begin{abstract}
Direct objects in the Dutch middlefield can either precede adverbs or follow them. This word order variation is traditionally labeled scrambling. Based on a corpus study of scrambling in spoken Dutch, we show that pronouns scramble almost categorically, whereas indefinite and definite objects scramble hardly at all. The observed effect of definiteness cannot be reduced to the influence of grammatical weight, in this way establishing both factors as independent determinants of word order variation. A closer investigation of proper noun objects shows that their position relative to the adverb is influenced by their animacy, length and stress. We argue that the ordering of elements in the Dutch middlefield is to be understood in terms of planning considerations on behalf of the speaker such that use of the unscrambled order buys him as much time for articulation of the direct object as possible.
\end{abstract}

Keywords: Dutch, scrambling, definiteness, weight, word order variation, speech planning

\section{Introduction}

The grammar of a language severely restricts the way in which a speaker may assemble his words into a sentence. At the same time, the grammar of many languages specifies certain configurations where the speaker is allowed to choose between different constructions. This even holds for languages which are said to obey a rather strict ordering of elements, such as English, and to a lesser extent other Germanic languages like German and Dutch. Such areas of constructional choice are important sources of grammatical variation. Traditionally, grammatical variation belongs to the domain of sociolinguistics (for synchronic variation) and historical linguistics (for diachronic variation), but with the increased availability and accessibility of large-scale corpora it has 
also considerably gained attention within theoretical linguistics. Accordingly, recent years have witnessed the appearance of many studies investigating the influence of different (grammatical) parameters, such as animacy, definiteness, and weight, on word order variation. For English, these include investigations into the genitive alternation (the king's palace vs. the palace of the king, e.g. Rosenbach 2005, Szmrecsanyi and Hinrichs 2008), particle verbs (he picked up the book vs. he picked the book up, e.g. Hawkins 1994, Gries 2003), the dative alternation (he gave the book to Mary vs. he gave Mary the book, e.g. Bresnan et al. 2005, Theijssen 2008) and heavy NP shift (e.g. Hawkins 1994, Wasow 2002).

In the present paper we investigate a type of word order variation, or constructional choice, in Dutch known as scrambling (sometimes also referred to as short scrambling, A-bar scrambling or object shift). Scrambling concerns the placement of the direct object with respect to an adverb, exemplified by the constructed examples in (1) and (2):

(1) Sonja heeft gisteren de kaas opgegeten.
Sonja has yesterday the cheese eaten
[2) Sonja heeft de kaas gisteren
Sonja has the cheese yesterday eaten
'Sonja ate the cheese yesterday.'

Two positions are available for direct objects with respect to adverbs in the Dutch middlefield (Mittelfeld): in what is traditionally called the unscrambled position the direct object follows the adverb, as in (1), whereas it precedes the adverb in the so-called scrambled position, illustrated in (2). ${ }^{1}$ Direct object scrambling is part of a set of scrambling phenomena (including also subjectobject, direct object-indirect object and focus scrambling) observed in a variety of languages such as the Germanic ones, Slavic, Turkic, Korean and Japanese (see e.g. the contributions to Karimi (ed.) 2003 and Sabel and Saito (eds) 2005 for discussion).

Scrambling in its various guises has received considerable attention in the theoretical linguistics literature. In the case of direct object scrambling this mainly concerns the syntactic question what kind of derivation underlies the scrambled order (see e.g. Thráinsson 2001, Putnam 2006 for an overview) ${ }^{2}$, and the semantic question which meanings are associated with different kinds of objects depending on their position relative to the adverb (e.g. de Hoop 1992, Diesing and Jelinek 1995, Ruys 2001). Surprisingly little work has been done on the manifestation of this phenomenon in naturally occurring language data, but see Yamashita (2003) for a corpus study on subject-object scrambling in Japanese and Kempen and Harbusch (2004), Heylen (2005), and Bader and Häussler (to appear) for variants of scrambling in German. In this paper we 
will try to further fill this lacuna by presenting a large-scale corpus study on direct object scrambling in spoken Dutch.

In van Bergen and de Swart (2009) we presented the results of a pilot corpus study in which we investigated the influence of definiteness and anaphoricity on direct object scrambling in spoken Dutch. Our main question concerned the alignment of object scrambling with the claims found in the theoretical literature on Germanic scrambling. In this literature two factors have been singled out that influence the scrambleability of objects: the referential type or definiteness of the direct object and its anaphoricity or, more generally, information structure (e.g. de Hoop 1992, Neeleman and Reinhart 1998, Meinunger 2000, Putnam 2006).

The discussion on definiteness generally makes a distinction between pronouns, indefinites, and definites. Much agreement exists on the scrambling behavior of the first two classes of direct objects. In Dutch, as in the other Germanic languages, pronouns scramble almost obligatorily, the unscrambled position being available only under specific stress patterns such as contrastive stress (e.g. de Hoop 1992, Bouma and de Hoop 2008, Meinunger 2000). Indefinite objects, by contrast, prefer to stay in the unscrambled position. Moreover, most researchers agree that when indefinite objects do scramble they undergo a shift in interpretation (Kerstens 1975, de Hoop 1992, Diesing and Jelinek 1995, but see Ruys 2001 for an alternative view). For definite NPs we have (at least) two theories with (partially) conflicting predictions on their scrambleability. According to Diesing and Jelinek (1995) referential definite objects scramble obligatorily, something that does not hold for non-referential definite objects. Van der Does and de Hoop (1998), on the other hand, strongly object to Diesing and Jelinek and claim that scrambling is truly optional for all definites (referential and non-referential ones), irrespective of the sentence or discourse context in which they occur. De Hoop (2003) further refines the claims of Van der Does and de Hoop (1998) by arguing that the scrambling of definite objects is influenced by their anaphoricity which is defined as previous mention in the discourse. According to the analysis proposed by de Hoop, anaphoric definites should scramble in two-thirds of the cases, whereas nonanaphoric ones only in half of the cases. Likewise, Jäger (1995) proposes for German object scrambling that full definite noun phrases with a so-called [+Topic] feature, which amounts to an anaphoric interpretation, scramble obligatorily (see Meinunger 2000 for a similar view).

On the basis of a sample of 2900 sentences from a corpus of spoken Dutch van Bergen and de Swart (2009) concluded that definiteness influences scrambling in a way different from what is generally assumed in the literature. We found a general decrease of scrambling along the definiteness hierarchy ( pronouns $>$ proper nouns $>$ definites $>$ indefinites): pronouns scrambled in $99 \%$ of the cases, proper nouns $53 \%$, definites only $12 \%$, and indefinites $2 \%$. 
Thus, contrary to what is suggested in the literature, definites do not scramble obligatorily, nor truly optionally. Instead, the only objects showing true variation in their scrambling behavior are proper nouns. The influence of anaphoricity also did not align with what is generally claimed in the literature. Although anaphoric definites scramble more often than non-anaphoric ones, they still do so only in $22 \%$ of the cases, remaining in the unscrambled position the other $78 \%$ of the times.

In the present paper we present the results of an extended corpus study on scrambling in spoken Dutch. We have almost tripled the sample size in comparison to the van Bergen and de Swart (2009) study in order to find answers to the following three questions:

1. Do our findings on the decrease of scrambling along the definiteness hierarchy hold up in a larger data set?

2. Can the definiteness effect on scrambling be reduced to a difference in grammatical weight? The general principle that short elements precede longer ones (see Hawkins 1994, Wasow 2002 for elaborate discussion), may be responsible for the pattern that pronouns, being short elements, appear before adverbs, whereas the opposite holds for full NPs, which are generally longer.

3. What factors condition the scrambling behavior of proper nouns? Should this variation be analyzed as random variation or can we identify certain factors (e.g. animacy, anaphoricity, stress) which influence the scrambling of these elements?

Based on a sample of well over 8000 examples we will show that claims in the literature about the scrambling behavior of different kinds of direct objects only partially hold in spoken language. We substantiate our previous findings that pronouns almost scramble categorically, whereas indefinite and definite objects scramble hardly at all. This bipartition in our data set may suggest that the observed pattern can be interpreted as an effect of grammatical weight. This is however not the case as a (regression) model based on definiteness distinctions is shown to have greater predictive power than one based on grammatical weight. This we take as evidence that effects of definiteness cannot be reduced to effects of weight, in contrast to what has been claimed in the literature (e.g. Hawkins 1994). In the one group of objects where true variation in scrambling is observed, those of the proper nouns, we will show that other factors that have been claimed to play a role in word order variation concerning arguments (including weight and animacy) have a clear effect on the relative ordering of arguments and adjuncts as well. The observed scrambling patterns in our set of spoken data are not fully in line with existing theoretical accounts of scrambling. We will argue that our findings can be explained by planning demands on behalf of the speaker. In particular, we will argue that the acces- 
sibility of arguments and a late-commitment strategy by the speaker (cf. Wasow 2002), i.e. trying to buy as much time for articulation as possible, can account for the distribution of scrambling in spoken Dutch.

This paper is organized as follows: in the next section we will give an overview of the factors that that are analyzed in our corpus study and discuss their hypothesized influence on scrambling in Dutch. Section 3 presents our corpus study of scrambling in spoken Dutch and contrasts the effects of definiteness and grammatical weight on it. Section 4 provides a closer look at proper nouns and investigates the factors that influence their position with respect to adverbs. In Section 5 we provide a general discussion of our findings and sketch our account in terms of planning. This discussion is followed by the conclusion in Section 6.

\section{Determinants of scrambling in Dutch}

In this section we present the factors that are analyzed in our corpus study and discuss their hypothesized influence on scrambling in Dutch. As stated in the introduction, in the first part of our study, we contrast the effects of definiteness and weight on object placement. In the second part, we focus on proper nouns, the one group of objects where variation is found, and consider the effects of animacy, weight, anaphoricity and stress on their position relative to adverbs.

\subsection{Definiteness}

Definiteness is often conceptualized in terms of a hierarchy ranging from pronouns to indefinite full NPs. Below we will discuss for each level on this hierarchy what has been claimed to be its behavior with respect to scrambling. We will see that for some types of elements there is disagreement in the literature. On the basis of the claims in the literature and our previous findings in van Bergen and de Swart (2009), we formulate our expectations for the corpus study presented in the later sections of this article.

- Pronouns: Different researchers agree on the influence of pronominality. In Dutch, as the other Germanic languages, unstressed pronouns scramble obligatorily. Pronouns can remain unscrambled in order when stressed or when heavy stress falls on the adverb (de Hoop 1992, Bouma and de Hoop 2008, Meinunger 2000). Accordingly, we expect the large majority of pronouns in our sample to scramble.

- Proper nouns: Claims about the scrambling behavior of proper nouns are limited to a few scattered remarks in the literature. Predictions of their 
scrambleability will be largely dependent on the specific analysis of proper nouns assumed (quantificational, definite descriptions), which will not concern us here. Distribution of claims on the positioning of proper nouns (in German) can be found in Meinunger (2000) who takes them to be 'relatively good base position occupants' (p. 80) yet to 'frequently undergo scrambling' (p. 88). Following van Bergen and de Swart (2009) we expect proper nouns to scramble at chance level.

- Definites: Predictions about the scrambling behavior of definites are largely dependent on the theory assumed. Diesing (1992; Diesing and Jelinek 1995) argues that only referential definite objects scramble obligatorily. Van der Does and de Hoop (1998), by contrast, state that scrambling is truly optional for all definites (referential and non-referential), irrespective of the sentence or discourse context in which they occur. Both approaches agree (and predict) that a substantial part of definite objects will scramble (assuming most definites are indeed referential in the case of Diesing's claims). This is not what we expect on the basis of our previous findings. According to those results scrambling will be strongly dispreferred.

- Indefinites: Much more agreement exists on the scrambling behavior of indefinite objects. Most researchers agree that these objects prefer to stay in the unscrambled position. This is also what we expect based on our previous study.

- Quantificational pronouns: Not much has been written on the behavior of quantificational pronouns like something, someone, and nothing. Meinunger (2000) claims that scrambling leads to ungrammaticalities in most cases, unless the objects are accented in a specific way. In our study we make a distinction between universally (strong, 'definite') quantified pronouns and existentially quantified (weak, 'indefinite') ones. According to Haspelmath (1997) (weak) quantificational pronouns are formally like pronouns, but functionally like noun phrases. If these elements behave as to their form we expect them to show an overwhelming tendency towards scrambling, just like regular pronouns. If, on the other hand, they behave according to their function, we expect them not to scramble very often. Moreover, if we find a difference within the class of quantificational pronouns we expect universally quantified ones to scramble more often than existentially quantified ones.

\subsection{Grammatical Weight}

The influence of grammatical weight or grammatical complexity on word order can be found in the preference to place longer/heavier constituents towards the end of the sentence (see, among others, Behaghel 1909, Hawkins 1994, Wasow 1997, 2002). This tendency is also referred to as short before 
long or the principle of end weight and seems to be independent of the way in which weight is conceptualized (Wasow 1997). Several corpus studies on Dutch found evidence for the fact that complex material is moved to the right periphery of sentences, but no opposite association between lighter constituents and positions earlier on in the sentence (Jansen and Wijnand 2004, van der Beek 2005, Bouma 2008). Correspondingly, we expect to find a strong preference for the unscrambled position in case of heavier objects. If lighter objects show a preference, it will be for the scrambled position.

\subsection{Animacy}

Animacy is well-known to have a profound effect on the ordering of arguments in sentences. From both typological and psycholinguistic studies there is clear evidence for the tendency to place animate arguments first in linear order, the so-called Animate First principle (e.g., Tomlin, 1986; Bock and Warren, 1985; Shridhar, 1988; McDonald et al. 1993, Van Nice and Dietrich, 2003; Branigan et al., 2008). For Dutch, van Bergen (2009) has found an effect of animacy on genitive ordering in a corpus of spoken Dutch relatively similar to that reported in English (Rosenbach 2002, 2005). Bouma (2008), in a corpus study on object fronting in Dutch, presents initial evidence that animate objects front more frequently than inanimate ones and that animate subjects discourage OVS order assuming that this may be because they want to occupy the initial position themselves. Accordingly, if animacy has an effect on the scrambling behavior of proper nouns, the number of scrambled animate proper nouns will be higher than that of scrambled inanimate ones.

\subsection{Information Structure: Anaphoricity and Stress}

Apart from definiteness many researchers also consider information structure, in particular anaphoricity, to have an effect on scrambling. For instance, de Hoop (2003) further refines the claims of Van der Does and de Hoop (1998) by arguing that the scrambling of definite objects is influenced by their anaphoricity which is defined as previous mention in the discourse (see also Jäger 1995 and Meinunger 2000 for German). For Dutch, Neeleman and Reinhart (1998) provide an analysis in which information structure and anaphoricity (D-linking in their terminology) play an important role. In their analysis they establish a connection between stress, D-linking, and scrambling. On the basis of the premises that (under neutral stress assignment) the object is destressed in scrambled position and that a noun phrase is destressed if and only if it is D-linked, they derive that scrambled direct objects are (very likely to be) D-linked. Given that, unlike indefinites, definite objects are almost standardly D-linked, it should follow that they are much more susceptible to scrambling, as the authors 
claim is indeed the case. Van Bergen and de Swart (2009) found that anaphoric definites indeed scramble more often than non-anaphoric ones, although they still exhibit an overall preference for the unscrambled position. Hence, in the present study, anaphoric proper nouns are expected to scramble more often than non-anaphoric ones. The opposite is expected for stressed proper nouns in comparison to their unstressed counterparts.

\section{Definiteness versus weight as predictor of scrambling}

\subsection{Data selection}

For this study we extended the data set used in van Bergen and de Swart (2009). Our sample has been extracted from the Corpus Gesproken Nederlands (Spoken Dutch Corpus, henceforth CGN). The CGN contains different types of contemporary Dutch speech, such as spontaneous face-to-face and telephone conversations, interviews, debates, radio shows and read-aloud books. Material is collected in the Netherlands (approximately two thirds) as well as in Flanders (about one third). All speech in this corpus has been orthographically transcribed, lemmatized and POS-tagged. Additionally, about 10 percent of the corpus has been annotated syntactically, which amounts to approximately 1 million words. From this syntactically annotated part we automatically extracted all transitive sentences in which a direct object either directly followed an adverb or directly preceded one, using TIGERSearch 2.1 (König et al. 2003). Instances of objects following or preceding multiple adverbs in one sentence were included in the data; sentences in which the object occurred in between two adverbs were left out as they could not be uniquely classified as scrambled or unscrambled. Moreover, adverbial prepositional phrases (e.g. in de tuin 'in the garden') and pronominal adverbs (e.g. er . . bij 'with it', hier ... op 'on this') were excluded. The remaining cases include adverbs of time (e.g. morgen 'tomorrow'), place (e.g. nergens 'nowhere'), frequency (e.g. soms 'sometimes'), degree (e.g. zeer 'very', helemaal 'totally'), manner (e.g. anders 'differently'), negation (e.g. niet 'not'), modal adverbs (e.g. misschien 'maybe', toch 'yet'), and combinations thereof. Clausal complements (hij zei gisteren dat hij ziek was 'yesterday, he said that he was ill') and fixed expressions were also removed from the data set as they do not allow for variation and hence are irrelevant to our research question. All remaining extracted sentences were manually checked and further noise was removed. In all sentences, the subject preceded both the object and the adverb. Our final sample contained a total of 8656 sentences, 3948 with a scrambled object and 4708 with an unscrambled object. ${ }^{3}$ We used R (R development core team 2008) for all statistical analyses. 
Table 1. A logistic regression model: definiteness

\begin{tabular}{lccr}
\hline Factor & $d f$ & estimate & $p$ \\
\hline DEFINITENESS & 5 & & \\
intercept (personal pronoun) & & 4.496 & 0 \\
proper noun & & -4.720 & 0 \\
univ pro & & -5.297 & 0 \\
definite NP & & -7.937 & 0 \\
exist pro & & -7.966 & 0 \\
indefinite NP & 8655 & -8.630 & \\
$n$ & 97.4 & & \\
$\%$ correct & 54.4 & & \\
$\%$ baseline & & & \\
\hline
\end{tabular}

\subsection{Logistic regression model: 6 levels of definiteness}

The definiteness of the direct object was subdivided into 6 levels (cf. Section 2.1):

1. [pers pro]: personal pronouns, clitics, reflexives and reciprocals;

2. [univ pro]: universally quantified pronouns;

3. [exist pro]: existentially quantified pronouns;

4. [PN]: personal names, place names and names of companies;

5. [def]: nominal objects preceded by a definite article, a demonstrative pronoun, a possessive pronoun, or a strong quantifier; ${ }^{4}$

6. [indef]: bare nominals, generic nouns and nominal objects preceded by a weak quantifier or an indefinite article.

Each case was classified by two annotators; disagreement between the annotators was resolved through discussion so that in the final annotation each object was provided with a unique definiteness feature.

In order to find out how well the definiteness of an object can be used as a predictor for the probability of scrambling, we used logistic regression (for an introduction to logistic regression, see e.g. Harrell 2001, Baayen 2008). ${ }^{5}$ A summary of this logistic regression model is given in Table 1. Our analysis shows a very strong correlation between the definiteness of the object and the position of the object relative to the adverb, which is highly significant: Loglikelihood ratio $X^{2}=10268.99 ; d f=5 ; p \approx 0$. Nagelkerke's $R^{2}$ is 0.929 , and the minimal adequate model has a very good classificatory power: $C=0.985$, $D_{\text {xy }}=0.969$.

First of all, we can see that this model predicts the scrambling behavior of direct objects correctly in $97.4 \%$ of the cases, which is a great improvement on the naïve model which chooses the most frequent (unscrambled) word order and hence achieves an accuracy of $54.4 \%$. A negative estimate points towards 


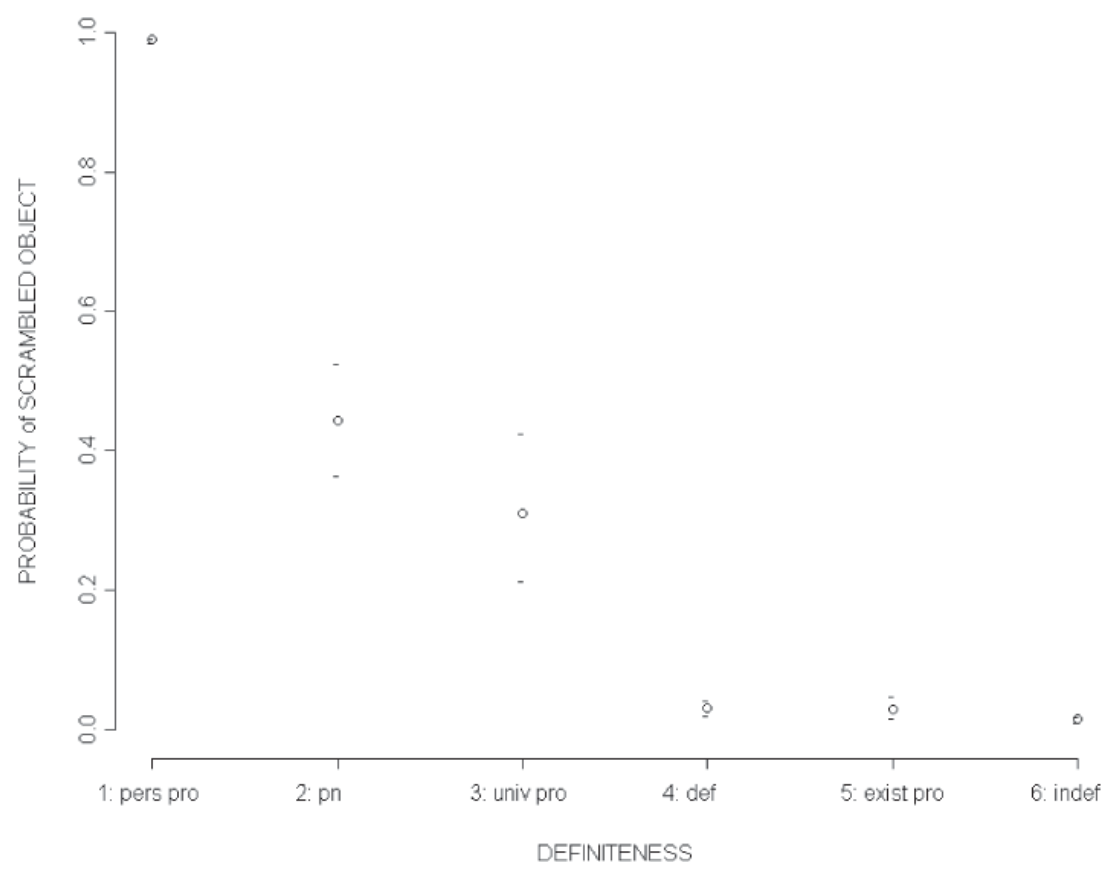

Figure 1. Probabilities of scrambled objects (with corresponding confidence intervals) per level of definiteness.

a lower probability of occurrence in the scrambled position; positive log odds ratios correspond to a higher scrambling probability. Personal pronouns are taken as the basic category (or intercept) and the estimates for the other definiteness levels are computed relative to the estimate of this basic level. For instance, the log odds ratio of -4.720 for proper noun objects means that the odds for a scrambled proper noun are (the inverse log of -4.720) 0.0089 times the odds for a scrambled pronominal object. In Figure 1, the probabilities of a scrambled object are given per definiteness class.

From these results, we can conclude that the findings of our previous smaller corpus study of scrambling in spoken Dutch (van Bergen and de Swart 2009) hold up in a larger data set (cf. question 1 raised in the introduction). The definiteness of the object turns out to be a very important predictor for scrambling, although its exact role differs largely from what is generally assumed in the literature (see van Bergen and de Swart 2009 for discussion). Personal pronouns almost always scramble while indefinite NPs hardly ever do. In contrast with the theoretical assumptions, definite NP objects pattern with indefinites in that they hardly occur in scrambled position. Only proper nouns do not show a clear preference for either the scrambled or the unscrambled position. Quanti- 
ficational pronouns, which received little discussion in earlier studies, show a preference for the unscrambled position, this preference being stronger for existentially than for universally quantified pronouns. From this we can tentatively conclude that quantificational pronouns seem to behave according to their functional similarity with full NPs rather than to their formal similarity with pronouns.

\subsection{Logistic regression model: weight}

In the previous section we have seen that a logistic regression model based on definiteness has a very good classificatory power: the model predicts the position of the object correctly in $97.4 \%$ of the cases. In the present section we address the question whether the observed patterns can receive an alternative (and better) interpretation in terms of grammatical complexity or weight. It has been argued (e.g. Hawkins 1994) that many of the factors governing word order variation (e.g. definiteness, givenness, animacy) can be reduced to the influence of grammatical complexity or weight, which can be found in the preference to place longer/heavier constituents towards the end of the sentence (cf. Section 2.2 above). Indeed, also in the present case it seems reasonable to suggest that the observed definiteness effects can be reduced to effects of weight: when we compare the elements that do scramble to the ones that do not, we find that the former (i.e. personal pronouns) are short whereas the latter ones (full NPs) are generally longer. In this section we will investigate whether the influence of definiteness on object scrambling in spoken Dutch is reducible to an effect of grammatical weight.

In order to calculate the correlation between definiteness and grammatical weight, we transformed the categorical variable of definiteness into an ordinal variable. The mutual ranking of the six levels of definiteness was determined by taking the order of the elements on the definiteness hierarchy (Aissen 2003) as a starting point. Universally and existentially quantified pronouns do not occur on Aissen's (2003) scale, and their position is hard to determine because of the difference in their formal and functional character. We decided to rank both types of quantificational pronouns according to their indefinite function rather than their pronominal form, in line with their scrambling behavior (cf. Section 3.2):

$\begin{array}{lllll}\text { (3) Definiteness scale: } & \text { pers pro }>\mathrm{PN}>\text { def } \mathrm{NP}>\text { univ pro }>\text { exist pro }> \\ \text { Rank } & 1 & 2 & 3 & 4 \\ & \text { indef NP } & & \\ & 6\end{array}$

Wasow $(1997,2002)$ has demonstrated that grammatical weight should be defined in terms of a gradient measure instead of a categorical one and that 
Table 2. A logistic regression model: object length

\begin{tabular}{lccc}
\hline Factor & $d f$ & estimate & $p$ \\
\hline Intercept & 1 & 7.701 & $<.000$ \\
Object length (log scale) & & -5.457 & $<.000$ \\
$n$ & 8656 & & \\
$\%$ correct & 89.4 & & \\
$\%$ baseline & 54.4 & & \\
\hline
\end{tabular}

different gradient measures highly correlate and hence are interchangeable. We therefore decided to measure the length of the object in number of characters. ${ }^{6}$

The ordinal definiteness variable correlates strongly with the length of the object: (Spearman's $\rho=0.81, p<0.0001$ ): higher ranked objects are shorter, whereas lower ranked objects are longer. ${ }^{7}$ The strong correlation between definiteness and weight could imply that the effect of definiteness on scrambling may in fact be an effect of object length. In order to determine whether the definiteness effect on scrambling can be considered an artifact of grammatical weight, we built a second logistic regression model, replacing definiteness with object length as the predictor of scrambling. As stated, the length of the object was measured in number of characters; values were log-transformed to reduce the effect of outliers.

The logistic regression model shows that there is a strong correlation between the object's length and its scrambling behavior which is highly significant: $\log$-likelihood ratio $X^{2}=7794.82 ; d f=1 ; p<0.0001$. Nagelkerke's $R^{2}$ is 0.794 , and the minimal adequate model has a very good classificatory power: $C=0.964, D_{\mathrm{xy}}=0.928$. A summary of the model is given in Table 2 .

The intercept has a positive value (7.701), which means that for the shortest objects the probability of scrambling is extremely high. The negative estimate of the factor object length is to be interpreted as follows: the longer the object, the smaller the probability of scrambling. This is graphically represented in Figure 2.

It has been argued that weight effects always depend on the relative weight of constituents rather than on the weight of a single one (Wasow 2002). Therefore, we also built a model that considers the relative weight of the object and the adverb as a predictor for scrambling to test whether we indeed find a stronger effect of short elements preceding long elements. The relative weight was measured by subtracting the adverb length from the object length (both measured in number of characters). The resulting logistic regression model (Table 3 ) shows that relative weight also strongly correlates with scrambling: this correlation is highly significant, $\log$-likelihood ratio $X^{2}=4963.54, d f=1, p \approx 0$. Nagelkerke's $R^{2}$ is 0.58 , and the classificatory power of this model is very good as well: $C=0.897, D_{\mathrm{xy}}=0.795$. The model's estimate of the intercept indicates 


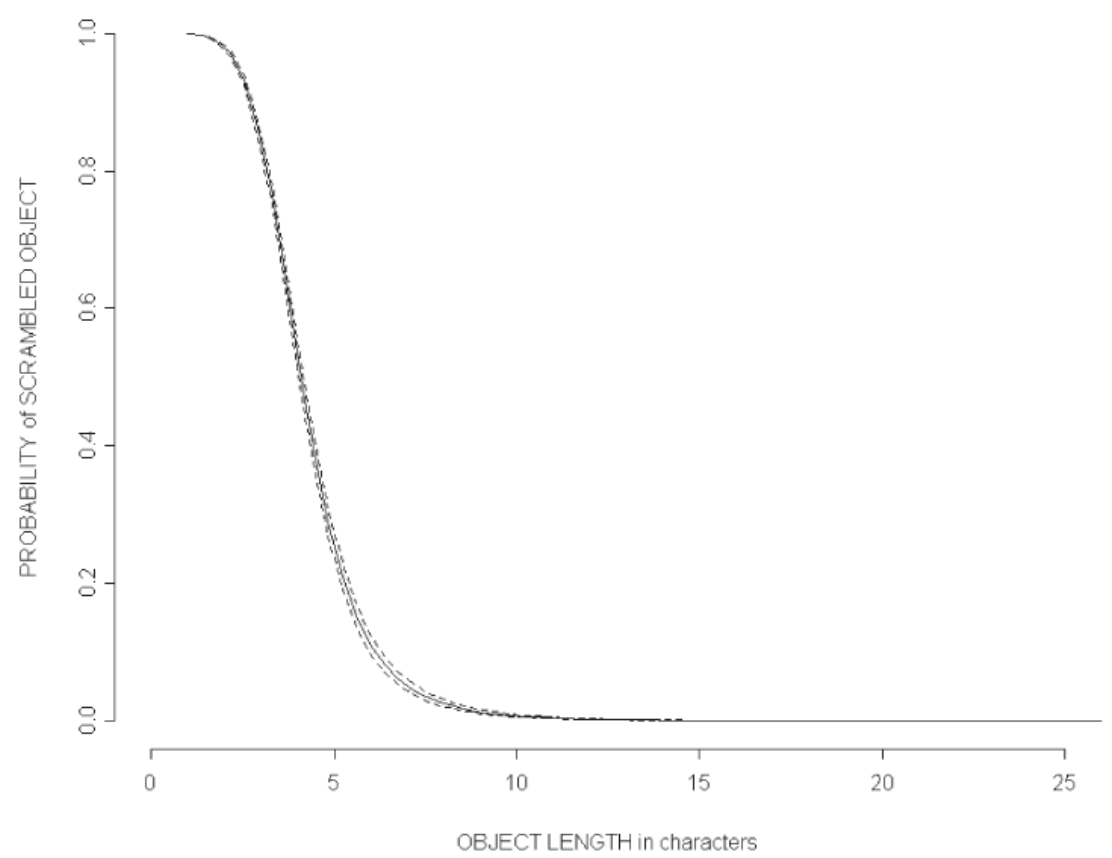

Figure 2. Probability of a scrambled object (with corresponding confidence intervals) by object length.

Table 3. Logistic regression model: relative weight

\begin{tabular}{lccc}
\hline Factor & $d f$ & estimate & $p$ \\
\hline Intercept & & -0.2897 & $<.000$ \\
Length difference (log scale) & 1 & -2.5324 & $<.000$ \\
$n$ & 8655 & & \\
\% correct & 81.2 & & \\
\% baseline & 54.4 & & \\
\hline
\end{tabular}

the log odds ratio when the length difference is zero, i.e., when the object and the adverb are equally long. The negative value indicates a (slight) preference for the unscrambled position. The negative estimate of the length difference factor indicates that the longer the direct object is relative to the adverb, the smaller the proportion of scrambling.

Comparing the models, we find a difference in predictive power: the relative weight model correctly predicts the position of the object $81.2 \%$ of the time, against a prediction accuracy of $89.4 \%$ for the absolute weight model. The 
difference between the models' likelihood ratios confirms that absolute object length is a better predictor than the relative length of object and adverb (residual deviance $X^{2}=2831.3$ ). Taking the length of the adverb into consideration does not improve the accuracy of the weight model. Instead, longer objects are more likely to occur to the right of the adverb, irrespective of the adverb's length. That is, we observe an absolute weight effect.

\subsection{Definiteness versus weight as scrambling predictor}

Both the definiteness model and the weight model show a highly significant effect on the scrambling behavior of direct objects, and both have a very high predictive power. The classification accuracy of the weight model is lower than that of the definiteness model, but with 1 degree of freedom the weight model is simpler than the definiteness model, which has 5 degrees of freedom. According to a likelihood ratio test, the gain in prediction accuracy outweighs the increase in degrees of freedom (residuals deviance $X^{2}(4)=2474.2, p \approx 0$ ). In other words, the definiteness model wins over the weight model. This strongly suggests that the definiteness effect cannot be reduced to an effect of weight (cf. question 2 of the introduction). In this way, we add to the existing evidence that, contra Hawkins (1994), effects of semantic/pragmatic features such as animacy, definiteness, and givenness cannot be considered epiphenomena of grammatical weight (Arnold et al. 2000, Rosenbach 2005). The remaining question is of course why definiteness would be a better predictor of scrambling than grammatical weight. To answer this question we investigated the accuracy difference between the definiteness and weight model more closely.

First, we compared the prediction accuracy of both models within each category of definiteness. The results are given in Table 4 .

Table 4. Prediction accuracy of both models within 6 definiteness groups

\begin{tabular}{|c|c|c|c|}
\hline & \multirow[t]{2}{*}{$n$} & \multicolumn{2}{|c|}{$\%$ predicted correctly } \\
\hline & & $\begin{array}{l}\text { definiteness } \\
\text { model }\end{array}$ & $\begin{array}{l}\text { weight } \\
\text { model }\end{array}$ \\
\hline Overall & 8656 & 97.4 & 89.4 \\
\hline Definiteness level & & & \\
\hline $\begin{array}{l}\text { personal pronouns } \\
\text { indefinite nouns }\end{array}$ & $\begin{array}{l}3809 \\
3169\end{array}$ & $\begin{array}{l}98.9 \\
98.4\end{array}$ & $\begin{array}{l}97.3 \\
92.7\end{array}$ \\
\hline definite nouns & 966 & 96.9 & 94.8 \\
\hline existentially quantified pronouns & 71 & 97.0 & 9.1 \\
\hline universally quantified pronouns & 497 & 69.0 & 31.0 \\
\hline proper nouns & 144 & 55.6 & 59.7 \\
\hline
\end{tabular}


Table 5. Prediction accuracy of both models within 6 length groups

\begin{tabular}{|c|c|c|c|}
\hline & \multirow[t]{2}{*}{$\mathrm{n}$} & \multicolumn{2}{|c|}{$\%$ predicted correctly } \\
\hline & & $\begin{array}{l}\text { definiteness } \\
\text { model }\end{array}$ & $\begin{array}{l}\text { weight } \\
\text { model }\end{array}$ \\
\hline Overall & 8656 & 97.4 & 89.4 \\
\hline \multicolumn{4}{|l|}{ Object length category } \\
\hline $1-3$ characters & 3870 & 99.1 & 93.6 \\
\hline 4-6 characters & 1384 & 92.0 & 57.6 \\
\hline 7-9 characters & 1128 & 95.4 & 95.1 \\
\hline 10-12 characters & 905 & 98.1 & 98.0 \\
\hline 13-15 characters & 582 & 99.5 & 99.5 \\
\hline$>15$ characters & 797 & 99.1 & 99.1 \\
\hline
\end{tabular}

Table 6. Prediction accuracy of the weight model for objects with a length of 4-6 characters

\begin{tabular}{|c|c|c|c|c|}
\hline & \multicolumn{2}{|c|}{ total } & \multicolumn{2}{|c|}{ predicted correctly } \\
\hline & $n$ & $(\%)$ & $n$ & $(\%)$ \\
\hline $4-6$ characters & 1384 & $(100)$ & 797 & $(58)$ \\
\hline \multicolumn{5}{|l|}{ Definiteness class } \\
\hline personal pronouns & 156 & (11) & 90 & $(58)$ \\
\hline indefinite nouns & 604 & (44) & 441 & (73) \\
\hline definite nouns & 172 & (12) & 150 & $(87)$ \\
\hline existentially quantified pronouns & 328 & (24) & 39 & (12) \\
\hline universally quantified pronouns & 66 & (5) & 45 & $(68)$ \\
\hline proper nouns & 58 & (4) & 32 & $(55)$ \\
\hline
\end{tabular}

When the data are split up according to the six levels of definiteness, we see the largest difference in accuracy within the category of existentially quantified pronouns: while the accuracy of the definiteness model is very high, the weight model performs extremely poorly within this category. Also for universally quantified pronouns the difference in accuracy between the models is quite large.

Next, we divided the objects into six categories according to their length and we compared the prediction accuracy of both models within each length category. ${ }^{8}$ The results are given in Table 5 .

The prediction accuracy of the two models is about equal in each category, except for the group of objects with a length of 4-6 characters: the prediction accuracy of the weight model is only little above chance level, whereas the definiteness model predicts $92 \%$ correctly. Looking at the types of objects that are correctly predicted by the weight model in this length group (Table 6), we see that prediction accuracy is again worst within the class of existentially 
quantified pronouns, which form a substantial part (about a quarter) of this length group.

We thus see that the main reason why the definiteness model outperforms the weight model lies with the existentially quantified pronouns: as these are relatively short they would be predicted to scramble much more often than they do in practice. Again, this can be seen as evidence that quantified pronouns do not behave according to their form, but rather to their function.

\section{A closer look at proper noun scrambling}

Proper noun objects show the most variation in their scrambling behavior. This group falls in between pronouns, which scramble almost always, and full NPs, which hardly ever scramble. Proper nouns do not show a clear preference for either the scrambled or the unscrambled position, so for this category there is some variation left to explain by predictors other than definiteness. In this section, we will therefore zoom in on the scrambling behavior of these types of objects, which should bring us the answer to the third and final question raised in the introduction to this paper.

\subsection{Statistical exploration}

In order to look for possible effects of the factors introduced in Section 2 (animacy, weight, anaphoricity, and stress), all sentences in our sample containing a proper noun object were annotated for these factors by two annotators; disagreement was resolved through discussion.

\subsubsection{Animacy}

We made a two-way animacy distinction: humans, non-human animals and names of organizations metonymically referring to humans were classified as animate; all other nouns (names of products, locations) were classified as inanimate. The frequency distribution of animate and inanimate objects over the two word orders is presented in Table 7. It can be seen from this table that animate objects scramble more often than inanimate objects; this difference is statistically significant $\left(X^{2}(1)=19.55, p<0.0001\right)$.

\subsubsection{Grammatical Weight}

Grammatical weight, defined as object length, was measured in number of characters, cf. Section 3.3. Figure 3 shows the length of the object in scrambled (left box) and unscrambled (right box) position. A U-test shows that the median length of scrambled objects $(3, I Q R=2)$ is significantly lower than the median length of objects in unscrambled position $(10, I Q R=9)$ : $W=619828.5, p \approx 0$. 
Table 7. Animacy effects on proper noun scrambling

\begin{tabular}{|c|c|c|c|c|c|c|}
\hline \multirow[t]{3}{*}{ ANIMACY } & \multicolumn{6}{|c|}{ POSITION } \\
\hline & \multicolumn{2}{|c|}{ Scrambled } & \multicolumn{2}{|c|}{ Unscrambled } & \multicolumn{2}{|c|}{ Total } \\
\hline & $n$ & $\%$ & $n$ & $\%$ & $\mathrm{n}$ & $\%$ \\
\hline Animate & 50 & $(61)$ & 32 & (39) & 82 & (100) \\
\hline Inanimate & 14 & (23) & 48 & $(77)$ & 62 & (100) \\
\hline TOTAL & 64 & (44) & 80 & $(56)$ & 144 & (100) \\
\hline
\end{tabular}

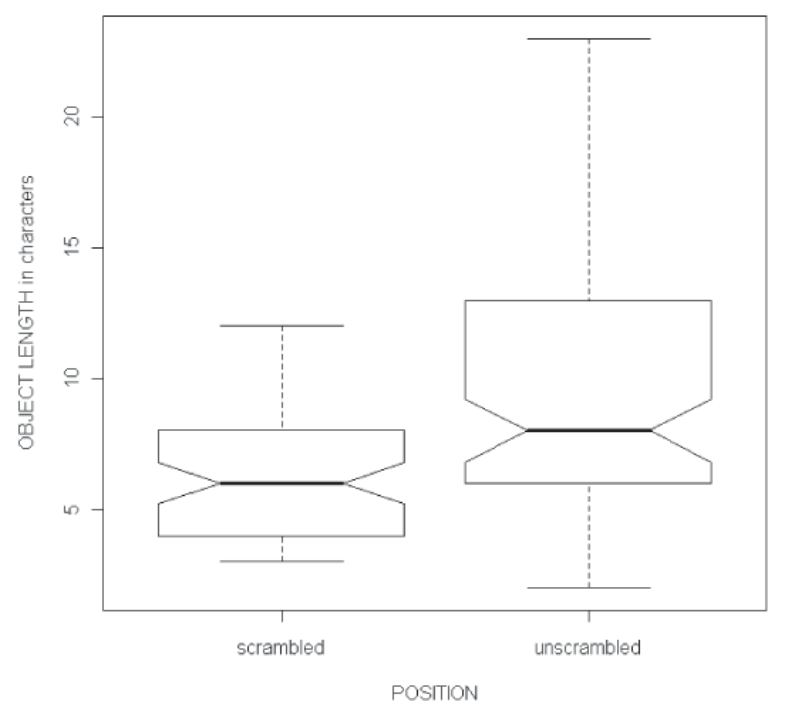

Figure 3. Boxplot of the object length in scrambled and unscrambled position

In other words, unscrambled proper nouns are generally longer than scrambled ones.

\subsubsection{Anaphoricity}

Following de Hoop's (2003: 205) definition, every proper noun was classified as anaphoric if it referred to an object previously mentioned in the discourse. Table 8 shows the frequency distribution of anaphoric and non-anaphoric proper nouns over the two word orders. There is a significant interaction between anaphoricity and scrambling $\left(X^{2}(1)=8.4216, p=0.004\right)$ : more than half of the anaphoric objects scramble, in comparison to only a third of the non-anaphoric ones. 
Table 8. Effects of anaphoricity on proper noun scrambling

\begin{tabular}{|c|c|c|c|c|c|c|}
\hline \multirow[t]{3}{*}{ ANAPHORICITY } & \multicolumn{6}{|c|}{ POSITION } \\
\hline & \multicolumn{2}{|c|}{ Scrambled } & \multicolumn{2}{|c|}{ Unscrambled } & \multicolumn{2}{|c|}{ Total } \\
\hline & $n$ & $\%$ & $n$ & $\%$ & $\mathrm{n}$ & $\%$ \\
\hline Anaphoric & 38 & $(58)$ & 27 & $(42)$ & 65 & (100) \\
\hline Non-anaphoric & 26 & (33) & 53 & (67) & 79 & (100) \\
\hline TOTAL & 64 & (44) & 80 & $(56)$ & 144 & $(100)$ \\
\hline
\end{tabular}

Table 9. Stress effects on proper noun scrambling

\begin{tabular}{|c|c|c|c|c|c|c|}
\hline \multirow[t]{3}{*}{ OBJECT STRESS } & \multicolumn{6}{|c|}{ POSITION } \\
\hline & \multicolumn{2}{|c|}{ Scrambled } & \multicolumn{2}{|c|}{ Unscrambled } & \multicolumn{2}{|c|}{ Total } \\
\hline & $\mathrm{n}$ & $\%$ & $\mathrm{n}$ & $\%$ & $\mathrm{n}$ & $\%$ \\
\hline Stressed & 33 & (33) & 67 & (67) & 100 & $(100)$ \\
\hline Unstressed & 31 & $(70)$ & 13 & (30) & 44 & $(100)$ \\
\hline TOTAL & 64 & (44) & 80 & (56) & 144 & (100) \\
\hline
\end{tabular}

\subsubsection{Stress}

Two annotators listened to every sentence with a proper noun object in the sample to determine whether or not an object was stressed. Table 9 shows that the majority of unstressed objects scramble, whereas the majority of stressed objects are in unscrambled position. This relation between stress and scrambling is significant $\left(X^{2}(1)=15.88, p<0.0001\right)$.

\subsection{A multifactorial regression model}

In the previous subsection we investigated the influence of the four factors on the scrambling of proper nouns separately. In natural language, however, these factors are all simultaneously involved in the choice for one word order over the other. To investigate the effects of all these factors on scrambling at the same time we built a logistic regression model, starting with a model including all possible factors introduced above and their interactions and then successively removing non-significant effects (stepwise backwards regression). The final model contains three factors: stress, animacy and object length. There were no significant interaction effects. The model has a likelihood ratio of $X^{2}=40.85, d f=3, p \approx 0$. Nagelkerke's $R^{2}$ is 0.331 ; the minimal adequate model has a good classificatory power: $C=0.802, D_{\mathrm{xy}}=0.604$. A summary of this logistic regression model is given in Table 10. 
Table 10. Logistic regression model of proper noun scrambling

\begin{tabular}{|c|c|c|c|}
\hline Factor & & estimate & $\mathrm{p}$ \\
\hline Intercept & & 1.9656 & $<0.05$ \\
\hline Object unstressed & & 1.41 & $<0.01$ \\
\hline Object inanimate & & -1.25 & $<0.01$ \\
\hline Object length (log scale) & & -1.10 & $<0.05$ \\
\hline $\mathrm{n}$ & 144 & & \\
\hline$\%$ correct & 74.3 & & \\
\hline$\%$ baseline & 55.6 & & \\
\hline
\end{tabular}

The prediction accuracy of the model is almost $75 \%$, which is about a third higher than the accuracy of the naïve model based on input frequency $(55.6 \%$ correct).

\subsection{Discussion}

The probabilistic model built in this section shows that when definiteness is indecisive, there is room for other factors to influence the scrambling behavior of objects (see Vogels 2009 for similar findings with respect to the placement of plural subjects in Dutch). The animacy effect suggests that animacy directly affects the linearization process (cf. also Kempen and Harbusch 2004 for German), in addition to reported indirect animacy effects on word order mediated through grammatical function assignment (Bock and Warren 1985). Moreover, the difference in scrambling behavior between animate and inanimate objects indicates that animacy has an absolute effect on word order, in the sense that animate arguments tend to occur early in the sentence as such, not relative to inanimate arguments in the same clause.

As for anaphoricity, even though anaphoric objects occur significantly more often in scrambled than in unscrambled position (cf. Table 8), it does not emerge as a significant scrambling predictor from the multifactorial model. This might be accounted for if we consider the relation between anaphoricity and stress (cf. Neeleman and Reinhart 1998), illustrated in Figure 4.

This figure shows that the number of stressed anaphoric objects is almost as high as the number of unstressed anaphoric objects (45\% vs. $55 \%$ respectively), whereas non-anaphoric objects are stressed in $90 \%$ of the cases. Considering that (a) non-anaphoric objects are almost always stressed, and (b) stressed objects scramble less often than unstressed objects, the fact that nonanaphoric objects scramble less often than anaphoric objects need not be expressed by a separate predictor in the model: this effect is already captured by the stress factor. Although the data show that there is a relation between anaphoricity and stress, the mapping is not as strict as claimed by Neeleman and 


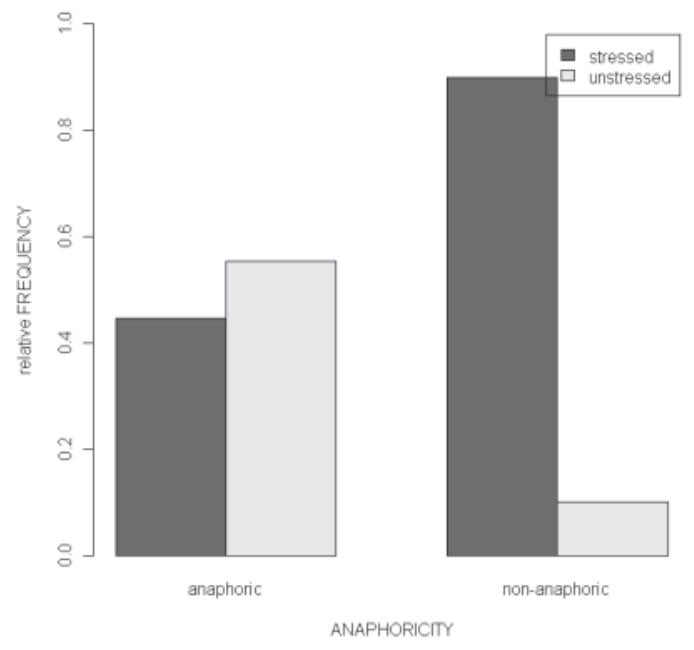

Figure 4. The relation between anaphoricity and stress

Reinhart (1998); the same holds for the relation between anaphoricity and position on the one hand, and stress and position on the other.

\section{General Discussion}

The picture of scrambling in spoken Dutch emerging from the discussion above is only partially in accordance with the one provided in the theoretical literature. Pronouns and indefinites are well behaved in this respect as they show a strong preference and dispreference for scrambling respectively, something which is generally observed in the literature, cf. Section 2. Definite direct objects, by contrast, do not behave as expected: they show a strong preference not to scramble, whereas it is generally argued that the opposite should hold. The fact that the scrambling of such elements is non-categorical makes it hard to capture it in terms of an absolute grammatical constraint. Instead, we argue for a functional interpretation of the data in terms of the planning of sentence production.

Given that scrambling is indeed a grammatical option generally available for speakers of Dutch, the question arises why language users make so little use of this possibility outside the domain of pronominal objects. The answer, we argue, has to be found in the way the speaker composes his utterances. More specifically, we adopt the late commitment approach advocated by Wasow (2002). Wasow analyzes the tendency of speakers to extrapose long/ heavy constituents as a mechanism to buy time for the precise formulation of 
such constituents; placing these elements at the end of the sentence reduces the amount of planning needed and allows more time to formulate and articulate thoughts. This approach can be naturally extended to our scrambling data. Like extraposition, scrambling, or rather not scrambling, provides the speaker with a mechanism to delay the expression of certain elements in the sentence. The unscrambled order allows him to buy some additional time for the formulation and planning of the object constituent by placing it after the adverb. In using the scrambled order the speaker gains time to plan the remainder of his utterance. Thus, under this account, we expect elements that require little planning to prefer the scrambled order and elements that require more planning to favor the unscrambled order. This is indeed seems to be the case.

The sentence production process involves (at least) grammatical and phonological/phonetic encoding. Schematically (and simplifying to a large extent) the former can be divided into the processes of lemma retrieval, (grammatical) function assignment, and linearization (e.g. Levelt 1989, Levelt et al. 1999, Ferreira \& Engelhardt 2006). It is generally assumed that the easier (and hence faster) a lemma can be retrieved, the more likely it is to occur earlier on in the realized string (i.e. the more prominent an item, the more prominent its position). ${ }^{9}$ Factors that are argued to influence this accessibility of lemmas are manifold and taken to include animacy, definiteness, discourse status, and predictability of items (e.g. Bock and Warren 1985, Levelt 1989, Prat-Sala and Branigan 2000). When applied to scrambling in Dutch, we would expect highly accessible items to occur earlier in the sentence, and hence to scramble, and less accessible items not to scramble.

This discussion of the influence of accessibility on sentence structure is (almost) exclusively limited to the linearization of two arguments (either subject and object or direct and indirect object). Given that our phenomenon involves the ordering of an argument and an adjunct, many of the features proposed to determine the accessibility of an item cannot be called upon as they are not applicable to adverbs (e.g. animacy and definiteness). Instead we have to resort to features relevant to pronouns, proper nouns, full NP (definite and indefinite) objects, and adverbs alike. We claim that these types of elements can be ordered in terms of their relative accessibility by referring to the type and size of the classes they are members of. This is summarized in Table 11. Pronouns are function words, whereas adverbs, proper nouns and NPs should be counted as content words. In contrast to content words, function words 'tend to be more accessible and easier to pronounce' (Clark and Wasow 1998: 210). Related to this distinction is a difference in frequency. Pronouns, adverbs and NPs all have a high class frequency (they occur very often in speech), whereas the class frequency of proper nouns is much lower. Yet, the first three class types do differ in class size (the number of unique class elements). Pronouns belong to a very small class; the class size of adverbs is already much bigger, just like 
Table 11. Relative predictability

\begin{tabular}{lllll}
\hline & PRONOUN & ADVERB & PN & NP \\
\hline Class type & Function & Content & Content & Content \\
Class frequency & High & High & Low & High \\
Class size $(\mathrm{n})$ & Low & Medium & Medium & High \\
Predictability $\left(1 / n_{\text {class }}\right)$ & High & Medium & Medium & Low \\
\hline
\end{tabular}

Table 12. Class frequency, class size and predictability based on 1000 sentences from the CGN

\begin{tabular}{lllll}
\hline & PRONOUN & ADVERB & PN & NP \\
\hline Class frequency & 2004 & 2062 & 353 & 3779 \\
Class size $(\mathrm{n})$ & 41 & 166 & 191 & 1270 \\
Predictability $\left(1 / n_{\text {class }}\right)$ & .024 & .006 & .005 & .0008 \\
\hline
\end{tabular}

that of proper nouns, but the class size of the latter two is not as big as that of NPs. This means that the search space for pronouns is relatively low in comparison to that of adverbs and proper nouns which in turn is relatively low in comparison to that of NPs. In other words, the predictability of specific items from these different classes and hence their accessibility decreases as one goes down the definiteness hierarchy.

The picture sketched in Table 11 is supported by the numbers in Table 12 . This table represents the class frequency, size and predictability of pronouns, adverbs and NPs in a sample of 1000 randomly selected sentences from the CGN.

The difference in predictability between these types of elements has important repercussions in the language production system. Due to their high accessibility in comparison to adverbs, pronouns will generally be available to the speaker before adverbs. Likewise, adverbs will generally be available earlier than NPs. These differences in accessibility can be directly related to the difference in scrambling behavior between the different types of objects. Given the low predictability of NPs, a speaker buys himself some additional time for the formulation and planning of this constituent by placing it after the adverb, which is relatively easier accessible. In case of a pronominal object, the speaker does not need this time and by placing it earlier on in the sentence he actually gains time to plan the remainder of the utterance. These scrambling patterns thus concur with a late commitment approach to language production.

The predictability of adverbs and proper nouns is almost equal, which is reflected in the almost optional nature of proper noun scrambling. When there is no clear predictability difference between two word classes in the way measured here, and hence no clear scrambling preference, factors that influence the accessibility within a word class (such as animacy and word length) may 
become manifest and influence the probability of scrambling, as shown in Section 4.

The account sketched above is compatible with both a radically incremental view on language production, i.e. nothing is planned ahead, as well as a limited incremental view, i.e. there is some planning. Recent experimental evidence (e.g. Ferreira and Swets 2002, Allum and Wheeldon 2007) suggests that language production is moderately incremental and that the scope of planning is subclausal corresponding roughly to the first constituent of a sentence. Although the precise incremental nature of the language production architecture is still a matter of debate, the general assumption is that it is capable of interleaving planning processes and articulation. This means that at a single point in time different parts of an utterance can be processed at different levels of encoding. Such parallel activation of information at different levels is required for the account of scrambling in spoken Dutch outlined above.

Our approach in terms of late commitment on behalf of the speaker concurs with the scrambling patterns found for pronominal and full NP objects. Due to their ambivalent nature, the picture is less clear for quantificational pronouns. Given that they functionally resemble full NPs but formally pronouns we could have expected them to pattern with either type of object. The fact that they behave like NP direct objects seems to suggest that in production more prominence is given to their functional status. Alternatively, the NP-like (nonpronounlike) behavior of quantificational pronouns may be argued to follow from the fact that scrambling of these elements results in a rather stark meaning contrast especially in the presence of logical adverbs (negation, quantifiers) where they acquire a wide-scope (specific) reading. Instead of using scrambling to express this meaning speakers may take recourse to a different kind of construction, like an expletive cleft (er is niemand die 'there is no one who'), which signals this meaning more clearly. A similar process could be envisaged for the low number of scrambling examples observed with indefinite NPs in general.

The low number of definite objects that scramble despite claims that this is a grammatical option raises the question whether we are witnessing a historical change. In other words, is NP-scrambling on its way out? Such a change is not without precedent in Germanic given the disappearance of scrambling in the history of English. Moreover, there is historical evidence that other types of scrambling than direct object scrambling are on decline in Dutch; see Hoeksema (2004) on focus scrambling. Given our account sketched above and following the Performance-Grammar Correspondence Hypothesis of Hawkins (2004), i.e. grammars conventionalize syntactic structures in proportion to their degree of preference in performance, we may expect a historical development to result in a state in which only pronoun scrambling is a grammatical option, making the language similar to the Scandinavian languages with respect to scrambling. 
Unfortunately, we lack historical evidence to substantiate a historical claim about direct object scrambling. Nevertheless, if scrambling is indeed on its way out, we might expect to find evidence for it by comparing spoken to written language, as the latter usually lags behind. Although we have not analyzed a comparable written data set, our data from spoken Dutch are coded for the distinction between spontaneous (e.g. conversations) and prepared (e.g. readaloud books) speech, which may be indicative. When we restrict ourselves to definites, we find that these objects scramble significantly more often in prepared speech than they do in spontaneous speech $\left(X^{2}(1)=9.3, p<0.01\right)$. It should, however, be noted that even in prepared speech definites have a strong tendency not to scramble (doing so only in $6 \%$ of the cases, as opposed to $2 \%$ in spontaneous speech). From this we may (tentatively) conclude that NP scrambling is underused both in informal and more formal Dutch; something which is compatible with the hypothesis that scrambling is on its way out. The fact that we find more scrambling in prepared speech may be seen as further evidence for our planning account sketched above. Given that writing puts less time pressure on the planning mechanism, this is the pattern that we anticipated.

The low frequency of NP scrambling in spoken Dutch may have its repercussions for the child acquiring this grammatical phenomenon. It is known from corpus counts and experiments on child production of scrambling that especially very young children (aged 2 and 3) show limited use of this mechanism (Barbier 2000, Schaeffer 2000a, 2000b). Generally it takes them up to the age of 5 to reach target-like (i.e. adult) behavior in experiments. Although the precise role of frequency on language acquisition is still a matter of debate (Tomasello 2003), the low number of NP scrambling in adult data may be one of the factors influencing the acquisition process.

\section{Conclusion}

In this article we have presented the results of a corpus study on scrambling in spoken Dutch. First, we have addressed the question in what way the definiteness of direct objects affects their scrambling behavior. Based on a sample of well over 8000 examples we have shown that existing claims in the literature only partially hold in spoken language, in this way substantiating our previous findings in van Bergen and de Swart (2009). More specifically, we found that pronouns scramble almost categorically, whereas indefinite and definite objects scramble hardly at all. In other words, the lower an object ranks in the definiteness hierarchy, the smaller its probability of occurring in scrambled position. Secondly, we considered whether the observed definiteness effect could be reduced to an effect of grammatical weight. Despite the substantial correlation between these two variables, we have argued that such a reduction 
is not justified as a regression model based on definiteness was shown to outperform a model with weight as the relevant predictor. In this way, we have added to the existing evidence that, contra Hawkins (1994), effects of semantic/ pragmatic features such as animacy, definiteness, and givenness cannot be considered epiphenomena of grammatical weight (cf. also Arnold et al. 2000, Rosenbach 2005). Finally, we presented a closer investigation of the factors influencing the scrambling behavior of proper noun objects. Scrambling in spoken Dutch can be characterized as an almost categorical phenomenon for most levels of definiteness. True word order variation is only found within the class of proper nouns. We have shown that their position relative to the adverb is influenced by animacy, length and stress, factors that have independently been claimed to play a role in word order variation.

The observed scrambling patterns in our set of spoken data are not fully in line with existing theoretical accounts of scrambling. Deviations are mainly caused by definite objects which showed a clear preference for the unscrambled position. We have argued that the ordering of elements in the Dutch middlefield should be understood in terms of planning considerations on behalf of the speaker and the accessibility of different types of objects. In particular, following Wasow (2002), we have adopted a late-commitment strategy, in which the speaker tries to buy as much time for articulation as possible by using the unscrambled order when the object has a rather low accessibility. Where accessibility is generally referred to when the ordering of two arguments is involved, we have shown that it can also be applied to the ordering of an argument with respect to an adjunct.

\section{Acknowledgements}

We would like to thank the editor-in-chief, two anonymous reviewers, Tom Wasow, Helen de Hoop, Ad Foolen, Lotte Hogeweg, Roeland van Hout, Sander Lestrade, Emar Maier, Kees de Schepper and Martine Zwets for useful comments on an earlier version of this article. Special thanks go to Jorrig Vogels and Peia Prawiro-Atmodjo, who helped to annotate large part of the data. The authors received financial support from the Netherlands Organization for Scientific Research (NWO) (grant 360-70-220, Animacy). The second author received additional support from the Netherlands Organization for Scientific Research (NWO) (grant 275-89-003, The Status of Hierarchies in Language Production and Comprehension).

\section{Bionotes}

Geertje van Bergen received her MA in 2006 from the Radboud University Nijmegen, the Netherlands. In December 2006 she started as a PhD student 
with her project "Animacy and word order in Dutch", a subproject of the NWO research project "Animacy". In her subproject she uses quantitative corpus data to investigate effects of multiple factors, especially animacy, on different types of word order variation in spoken Dutch. E-mail: g.vanbergen@let.ru.nl

Peter de Swart received his PhD in 2007 from the Radboud University Nijmegen, the Netherlands, with a dissertation on the cross-linguistic influence of semantic features, in particular animacy, on the marking of direct objects. Until the end of 2008 he continued his research as a postdoctoral researcher at the same university. Since January 2009 he is a postdoctoral researcher at the Center for Language and Cognition Groningen of the University of Groningen, the Netherlands. In his current project "The status of hierarchies in language production and comprehension" he investigates the role of hierarchies of individuation in grammar and language use. E-mail: p.j.f.de.swart@rug.nl

\section{Notes}

1. This terminology can be traced back to the traditional theoretical assumption (as advocated in much of the generative syntactic approaches; but see note 2) that the position to the right of the adverb is the basic (or underlying) position of the direct object. The position to the left of the adverb is considered to be derived by means of some sort of movement operation.

2. There is considerable discussion among syntacticians whether the direct object is generated in the position to the left of the adverb (Neeleman 1994) or has moved there from its base position inside the VP, which brings along the question what kind of movement it has undergone (for Dutch, Bennis and Hoekstra 1984 argued it should be A movement, whereas Vanden Wyngaerd 1989 takes it to be A-bar movement; see also Putnam 2006 for an overview of this discussion concerning scrambling in the Germanic languages in general). As this question is not directly relevant to our purposes, we will remain agnostic about it.

3. Van de Cruys (2005: 80) found an almost identical relative distribution of scrambled and unscrambled direct objects in the Flemish part of the CGN.

4. The strength of a quantifier was determined through the admissibility of occurrence in a presentational context (er zijn ... 'there are ...'), a context only allowed for weak elements (Milsark 1979).

5. We used the Design package (Harrell 2008) for the logistic regression modelling.

6. The reason we did not measure object length in number of words is that Dutch is a compoundfriendly language, as a result of which two words can differ greatly in character length, making character length a more robust measure.

7. If existentially and universally quantified pronouns are ranked according to their form, that is, if placed in between pronouns and proper nouns on the definiteness scale, we find a slightly higher correlation between definiteness and weight (Spearman's $\rho=0.84, p<0.0001$ ).

8. This transformation of object length from a gradient into a discrete variable is for exploratory purposes only. The accuracy difference between definiteness and weight is irrespective of the discrete categories introduced here, as the definiteness model was already shown to outperform the gradient weight model in the previous section.

9. This process may or may not be mediated through grammatical function assignment (Branigan et al. 2008); see also Section 4. 


\section{References}

Aissen, Judith. 2003. Differential object marking: Iconicity vs. economy. Natural Language and Linguistic Theory 21(3). 435-483.

Allum, Paul H. \& Linda R. Wheeldon 2007. Planning Scope in Spoken Sentence Production: The Role of Grammatical Units. Journal of Experimental Psychology: Learning, Memory, and Cognition 33(4). 791-810.

Arnold, Jennifer E., Thomas Wasow, Anthony Losongco \& Ryan Ginstrom. 2000. Heaviness vs. newness: the effects of structural complexity and discourse status on constituent ordering. Language 76(1). 28-55.

Baayen, R. Harald. 2008. Analyzing linguistic data. A practical introduction to statistics using $R$. Cambridge: Cambridge University Press.

Bader, Markus \& Jana Häussler. to appear. Word order in German: A corpus study. Lingua.

Barbier, Isabella. 2000. An experimental study of scrambling and object shift in the acquisition of Dutch. In Susan M. Powers and Cornelia Hamann (eds.), The acquisition of scrambling and cliticization, 41-69. Dordrecht: Kluwer Academic Publishers.

Behaghel, Otto. 1909. Beziehungen zwischen Umfang und Reihenfolge von Satzgliedern. Indogermanische Forschungen 25. 110-142.

Bennis, Hans \& Teun Hoekstra. 1984. Gaps and Parasitic Gaps. The Linguistic Review 4(1). 29 87.

van Bergen, Geertje. 2009. The Dutch genitive alternation. Paper presented at the Optimal Communication Colloquium, Radboud University Nijmegen, January 21.

van Bergen, Geertje \& Peter de Swart. 2009. Definiteness and scrambling in Dutch: where theory meets practice. In: A. Schardl, M. Walkow and M. Abdurrahman (eds), Proceedings of NELS 38 , 113-124. Amherst, MA: GLSA.

Bock, Kathryn \& Richard K. Warren. 1985. Conceptual accessibility and syntactic structure in sentence formulation. Cognition 21(1).47-67.

Bouma, Gerlof. 2008. Starting a sentence in Dutch. A corpus study of subject- and object-fronting. $\mathrm{Ph} . \mathrm{D}$ dissertation, University of Groningen.

Bouma, Gerlof \& Helen de Hoop 2008. Unscrambled pronouns in Dutch. Linguistic Inquiry 39 (4). 669-677.

Branigan, Holly P., Martin J. Pickering \& Mikihiro Tanaka. 2008. Contributions of animacy to grammatical function assignment and word order production. Lingua 118(2). 172-189.

Bresnan, Joan, Anna Cueni, Tatiana Nikitina \& Harald Baayen. 2005. Predicting the dative alternation. In Gosse Bouma, Irene Kraemer \& Joost Zwarts (eds), Cognitive foundations of interpretation, 69-94. Amsterdam: Royal Netherlands Academy of Science.

Clark, Herbert H. \& Thomas Wasow. 1998. Repeating words in spontaneous speech. Cognitive Psychology 27(3). 201-42.

van de Cruys, Tim. 2005. Between VP Adjuncts and Second Pole in Dutch. A corpus based survey regarding the complements between VP adjuncts and second pole in Dutch. In Ton van der Wouden, Michaela Poss, Hilke Reckman \& Crit Cremers (eds.), Computational Linguistics in the Netherlands 2004: Selected papers from the fifteenth CLIN meeting, 75-88. Utrecht: LOT Publications.

Diesing, Molly. 1992. Indefinites. Cambridge: MIT Press.

Diesing, Molly \& Eloise Jelinek. 1995. Distributing Arguments. Natural Language Semantics 3(2). 123-176.

van der Does, Jaap \& Helen de Hoop. 1998. Type-shifting and scrambled definites. Journal of Semantics 15(4). 393-416.

Ferreira, Fernanda \& Paul Engelhardt. 2006. Syntax and production. In M. A. Gernsbacher \& M. Traxler (Eds.), Handbook of Psycholinguistics, 61-91. Oxford, UK: Elsevier Inc. 
Ferreira, Fernanda \& Benjamin Swets. 2002. How Incremental Is Language Production? Evidence from the Production of Utterances Requiring the Computation of Arithmetic Sums. Journal of Memory and Language 46(1). 57-84.

Gries, Stefan Th. 2003. Multifactorial analysis in corpus linguistics: a study of particle placement. New York: Continuum Press.

Harrell, Frank E. Jr. 2001. Regression modeling strategies. Berlin: Springer.

Harrell, Frank E. Jr. 2008. Design: Design Package. R package version 2.1-2. http://biostat. mc.vanderbilt.edu/s/Design, http://biostat.mc.vanderbilt.edu/rms.

Haspelmath, Martin. 1997. Indefinite pronouns. Oxford: Clarendon Press.

Hawkins, John A. 1994. A performance theory of order and constituency. Cambridge: Cambridge University Press.

Hawkins, John A. 2004. Efficiency and complexity in grammars. Oxford: Oxford University Press. Heylen, Kris. 2005. A Quantitative Corpus Study of German Word Order Variation. In Stephan Kepser en Marga Reis (eds.), Linguistic evidence: empirical, theoretical and computational perspectives, 241-264. Berlin, Mouton de Gruyter.

Hoeksema, Jack. 2004. In het minst: eigenschappen en ontwikkeling van een negatief-polaire uitdrukking en de neergang van focus scrambling. TABU: Bulletin voor Taalwetenschap 33(1/2). 27-50.

de Hoop, Helen. 1992. Case configuration and Noun Phrase interpretation. Ph.D. dissertation, University of Groningen.

de Hoop, Helen. 2003. Scrambling in Dutch: optionality and optimality. In Simin Karimi (ed.), Word order and scrambling, 201-216. Oxford: Blackwell.

Jäger, Gerhard. 1995. Topic, scrambling, and aktionsart. In Inga Kohlhof, Susanne Winkler \& Hans Bernhard Drubig (eds.), Proceedings of the Göttingen Focus Workshop, 19-34. Tübingen, Arbeitspapiere des SFB 340 'Sprachtheoretische Grundlagen für die Computerlinguistik'.

Karimi, Simin (ed.). 2003. Word Order and Scrambling. Oxford: Blackwell.

Kempen, Gerard \& Karin Harbusch. 2004. A corpus study into word order variation in German subordinate clauses: Animacy affects linearization independently of grammatical function assignment. In Thomas Pechmann \& Christoph Habel (eds.), Multidisciplinary approaches to language production, 173-181. Berlin: Mouton De Gruyter.

Kerstens, Johan. 1975. Over afgeleide structuur en de interpretatie van zinnen. Ms. University of Amsterdam.

König, Esther, Wolfgang Lezius \& Holger Voormann. 2003. TIGERSearch 2.1 User's Manual. IMS, University of Stuttgart.

Levelt, Willem J. M. 1989. Speaking: From intention to articulation. Cambridge, MA: MIT Press.

Levelt, Willem J. M., Ardi Roelofs \& Antje S. Meyer. 1999. A theory of lexical access in speech production. Behavioral and Brain Sciences 22(1). 1-75.

McDonald, Janet, Kathryn Bock \& Michael Kelly. 1993. Word and world order: semantic, phonological and metrical determinants of serial position. Cognitive Psychology 25(2). 188-230.

Meinunger, André. 2000. Syntactic aspects of topic and comment. Amsterdam: John Benjamins.

Milsark, Gary. 1974. Existential sentences in English. PhD. dissertation, Cambridge, Mass: MIT Press. Published as Outstanding dissertations in linguistics 19. New York: Garland Publishers.

Neeleman, Ad. 1994. Scrambling as a D-structure phenomenon. In Norbert Corver \& Henk C. van Riemsdijk (eds.), Studies on Scrambling: movement and non-movement approaches to freeword-order phenomena, 387-429. Berlin: Mouton de Gruyter.

Neeleman, Ad \& Tanya Reinhart. 1998. Scrambling and the PF interface. In Miriam Butt and Wilhelm Geuder (eds.), The projection of arguments, 309-353. Stanford, CA: CSLI Publications.

Prat-Sala, Mercè \& Holly P. Branigan. 2000. Discourse constraints on syntactic processing in language production: a cross-linguistic study in English and Spanish. Journal of Memory and Language 42(2). 168-182. 
Putnam, Michael T. 2007. Scrambling and the Survive Principle. Amsterdam/Philadelphia: John Benjamins.

R Development Core Team. 2008. R: A language and environment for statistical computing. $\mathrm{R}$ Foundation for Statistical Computing, Vienna, Austria. ISBN 3-900051-07-0, URL http://www. R-project.org.

Rosenbach, Anette. 2005. Animacy versus weight as determinants of grammatical variation in English. Language 81(3). 613-644.

Ruys, Eddy. 2001. Dutch Scrambling and the Strong-Weak Distinction. Journal of Comparative Germanic Linguistics 4(1). 39-67.

Sabel, Joachim and Mamoru Saito (eds.). 2005. The free word order phenomenon. Berlin/New York: Mouton de Gruyter.

Schaeffer, Jeannette C. 2000a. The Acquisition of Direct Object Scrambling and Clitic Placement: Syntax and Pragmatics. Amsterdam/Philadelphia: John Benjamins.

Schaeffer, Jeannette. 2000b. Scrambling and specificity in Dutch child language. In Susan M. Powers \& Cornelia Hamann (eds.), The acquisition of scrambling and cliticization, 71-93. Dordrecht: Kluwer Academic Publishers.

Szmrecsanyi, Benedikt \& Lars Hinrichs. 2008. Probabilistic determinants of genitive variation in spoken and written English: a multivariate comparison across time, space, and genres. In Terttu Nevalainen, Irma Taavitsainen, Päivi Pahta \& Minna Korhonen (eds.), The dynamics of linguistic variation: corpus evidence on English past and present, 291-309. Amsterdam: John Benjamins.

Theijssen, Daphne. 2008. Using the ICE-GB Corpus to model the English dative alternation. Proceedings of the Aston Postgraduate Conference on Corpus Linguistics. http://lands.let.ru.nl/ $\sim$ daphne/publications/ (accessed 10 July 2009).

Thráinsson, Höskuldur. 2001. Object Shift and Scrambling. In Mark Baltin \& Chris Collins (eds.), The handbook of contemporary syntactic theory, 148-202. Oxford: Blackwell.

Tomasello, Michael. 2003. Constructing a language. A usage-based theory of language acquisition. Cambridge/London: Harvard University Press.

Vanden Wyngaerd, Guido. 1989. Object shift as an A-movement rule. MIT Working Papers in Linguistics 11. 256-271.

Vogels, Jorrig. 2009. Topic first or Subject first: A corpus study of the position of bare and definite plural subjects in Dutch. MA thesis, Radboud University Nijmegen.

Wasow, Thomas. 1997. Remarks on Grammatical Weight. Language Variation and Change 9(1). $81-105$.

Wasow, Thomas. 2002. Postverbal behavior. Stanford, CA: CSLI Publications.

Yamashita, Hiroko. 2002. Scrambled sentences in Japanese: linguistic properties and motivations for production. Text 22(4). 597-633. 